\title{
Light relic neutralinos
}

\section{Stefano Scopel*}

Dipartimento di Fisica Teorica, Università di Torino and INFN, Sez. di Torino

Via P. Giuria 1, I-10125 Torino, Italy

E-mail: iscopel@to.infn.it'

ABStRaCt: The relic abundance and the scalar cross-section off nucleon for light neutralinos (of mass $m_{\chi}$ below about $45 \mathrm{GeV}$ ) are evaluated in an effective MSSM model with R-parity conservation and without GUT-inspired relations among gaugino masses. It is shown that these neutralinos may provide a sizeable contribution to the matter density in the Universe $\Omega_{C D M}$. By requiring that its relic abundance does not exceed the upper bound on $\Omega_{C D M}$ based on the new WMAP data, a lower bound on the neutralino mass $m_{\chi} \gtrsim 6 \mathrm{GeV}$ is derived. These light neutralinos can also produce measurable effects in WIMP direct detection experiments, and in particular could explain the modulation result recently confirmed by DAMA. Uncertainties in direct detection calculations due to the modeling of the WIMP velocity distribution function are also discussed.

\section{Introduction}

Most works on relic neutralinos consider supersymmetric schemes with a unification assumption for the gaugino masses $M_{i}(i=1,2,3)$ at the GUT scale $M_{G U T} \sim 10^{16} \mathrm{GeV}$. This hypothesis implies that at at the electroweak scale, $M_{E W} \sim 100 \mathrm{GeV}$, the following relation holds:

$$
M_{1}=\frac{5}{3} \tan ^{2} \theta_{W} M_{2} \simeq 0.5 M_{2} .
$$

where $\theta_{W}$ is the Weinberg angle. The most direct consequence of Eq. (1.1. 1) is that the present lower bound on the chargino mass, $m_{\chi_{ \pm}} \gtrsim 100 \mathrm{GeV}$, implies a lower bound on the the neutralino mass, $\left.m_{\chi} \gtrsim 30 \mathrm{GeV}[]_{1}^{1}\right]$. However, no model-independent direct limits on the neutralino mass exist at present, so that, if Eq. (1) (1) i $\left.1_{1}^{\prime}\right)$ is relaxed, neutralinos lighter than $30 \mathrm{GeV}$ could be compatible with observations, with important phenomenological implications.

In the present talk I will discuss the properties of relic neutralinos in an effective Minimal Supersymmetric extension of the Standard Model (MSSM) where the GUT relation

${ }^{*}$ Speaker. 
of Eq. (1.1.1.1.1) is relaxed, with particular emphasis on light neutralinos. More details, as well as a list of references to previous papers where supersymmetric schemes without gaugino masses unification have been considered in connection with relic neutralinos, can be found

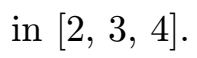

\section{Effective MSSM without gaugino unification}

We employ an effective MSSM scheme (effMSSM) at the electroweak scale, defined in terms of a minimal number of parameters, only those necessary to shape the essentials of the theoretical structure of MSSM and of its particle content. The assumptions that we impose at the electroweak scale are: a) all squark soft-mass parameters are taken degenerate: $m_{\tilde{q}_{i}} \equiv m_{\tilde{q}} ; \mathrm{b}$ ) all slepton soft-mass parameters are taken degenerate: $m_{\tilde{l}_{i}} \equiv m_{\tilde{l}}$; c) all trilinear parameters are set to zero except those of the third family, which are defined in terms of a common dimensionless parameter $A: A_{\tilde{b}}=A_{\tilde{t}} \equiv A m_{\tilde{q}}$ and $A_{\tilde{\tau}} \equiv A m_{\tilde{l}}$. As a consequence, the supersymmetric parameter space consists of the following independent parameters: $M_{2}, \mu, \tan \beta, m_{A}, m_{\tilde{q}}, m_{\tilde{l}}, A$ and $R \equiv M_{1} / M_{2}$. In the previous list of parameters we have denoted by $\mu$ the Higgs mixing mass parameter, by $\tan \beta$ the ratio of the two Higgs v.e.v.'s and by $m_{A}$ the mass of the CP-odd neutral Higgs boson.

The neutralino is defined as the lowest-mass linear superposition of bino $\tilde{B}$, wino $\tilde{W}^{(3)}$ and of the two higgsino states $\tilde{H}_{1}^{\circ}, \tilde{H}_{2}^{\circ}$ :

$$
\chi \equiv a_{1} \tilde{B}+a_{2} \tilde{W}^{(3)}+a_{3} \tilde{H}_{1}^{\circ}+a_{4} \tilde{H}_{2}^{\circ} .
$$

Due to well-known properties of the neutralino and chargino mass matrices, one has that: a) for $\mu \gg M_{1}, M_{2}$ the neutralino mass is determined by the lightest gaugino mass parameter: $m_{\chi} \simeq \min \left(M_{1}, M_{2}\right)$, while the lightest chargino mass is set by $M_{2}: m_{\chi^{ \pm}} \simeq M_{2}\left(M_{1}\right.$ does not enter the chargino mass matrix at tree-level); b) for $\mu \ll M_{1}, M_{2}$ both the neutralino and the chargino masses are primarily set by the Higgs mixing parameter: $m_{\chi} \simeq \mu \simeq m_{\chi^{ \pm}}$.

LEP data put a stringent lower bound on the chargino mass: $m_{\chi^{ \pm}} \gtrsim 103 \mathrm{GeV}$, which converts into lower bounds on $M_{2}$ and $\mu: M_{2}, \mu \gtrsim 103 \mathrm{GeV}$. This implies a lower bound on the neutralino mass of the order of about $50 \mathrm{GeV}$ in the standard effMSSM, where the GUT relation of Eq.(i1.1i) holds. On the contrary, the neutralino mass may be smaller when $M_{1} \ll M_{2}$, thus for small values of the parameter $R$.

We are now interested in the phenomenology of light neutralinos, therefore we consider values of $R$ lower than its GUT value: $R_{G U T} \simeq 0.5$. For definiteness we will consider the range: $0.01-0.5$. By diagonalizing the usual neutralino mass matrix in the approximation $M_{1}<<M_{2}, \mu$, it turns out that light neutralinos have a dominant bino component; a deviation from a pure bino composition is mainly due to a mixture with $\tilde{H}_{1}^{\circ}, i$ e. $\left|a_{1}\right|>>$ $\left|a_{3}\right|>>\left|a_{1}\right|,\left|a_{4}\right|$. For the ratio $\left|a_{3}\right| /\left|a_{1}\right|$ one finds

$$
\frac{\left|a_{3}\right|}{\left|a_{1}\right|} \simeq \sin \theta_{W} \sin \beta \frac{m_{Z}}{|\mu|} \lesssim 0.42 \sin \beta
$$

where $m_{Z}$ is the mass of the $Z$ boson, and in the last step we have taken into account the experimental lower bound $\mu \gtrsim 100 \mathrm{GeV}$. 
In our numerical analysis we have varied the MSSM parameters within the following ranges: $1 \leq \tan \beta \leq 50,100 \mathrm{GeV} \leq|\mu|, M_{2}, m_{\tilde{q}}, m_{\tilde{l}} \leq 1000 \mathrm{GeV}, \operatorname{sign}(\mu)=-1,1,90 \mathrm{GeV} \leq$ $m_{A} \leq 1000 \mathrm{GeV},-3 \leq A \leq 3,0.01 \leq R \leq 0.5$. This range for $R$, implemented with the experimental lower limit on $M_{2}$ of about $100 \mathrm{GeV}$, implies that the lower bound on the neutralino mass can be moved down to few GeV's for $R \sim 0.01$.

We then implemented the following experimental constraints: accelerators data on supersymmetric and Higgs boson searches (CERN $e^{+} e^{-}$collider LEP2 [9i] and Collider

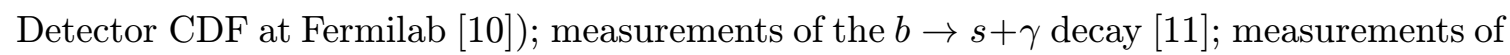
the muon anomalous magnetic moment $a_{\mu} \equiv\left(g_{\mu}-2\right) / 2$ [1] $\overline{1}_{-1}$, (the range $-160 \leq \Delta a_{\mu} \cdot 10^{11} \leq$ 680 is used here for the deviation of the current experimental world average from the theoretical evaluation within the Standard Model; for the derivation see Ref. [류] $)$.

\section{Cosmological lower bound on $m_{\chi}$}

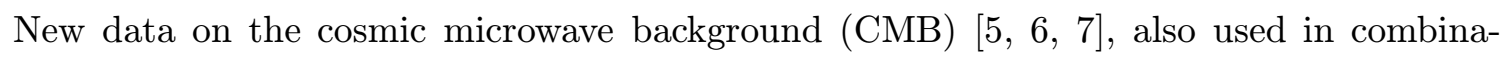
tion with other cosmological observations, are progressively narrowing down the ranges of the relic abundances for matter $\left(\Omega_{m} h^{2}\right)$ and for some of its constituents: neutrinos $\left(\Omega_{\nu} h^{2}\right)$ and baryons $\left(\Omega_{b} h^{2}\right)$. As a consequence also the range of $\Omega_{C D M} h^{2}$ is reaching a unprecedented level of accuracy. Allowing for a $2-\sigma$ range in $\Omega_{C D M} h^{2}$, one obtains [i3i] : $\left(\Omega_{C D M} h^{2}\right)_{\min }=0.095 \leq \Omega_{\chi} h^{2} \leq\left(\Omega_{C D M} h^{2}\right)_{\max }=0.131$. These are the values we will use here. However, one should cautiously still be open to some possible changes in these values as new cosmological observational data will accumulate in the future.

The neutralino relic abundance is given by

$$
\Omega_{\chi} h^{2}=\frac{x_{f}}{g_{\star}\left(x_{f}\right)^{1 / 2}} \frac{3.3 \cdot 10^{-38} \mathrm{~cm}^{2}}{<\widetilde{\sigma_{a n n} v}>}
$$

where $\left\langle\widetilde{\sigma_{\text {ann }} v}>\equiv x_{f}\left\langle\sigma_{\text {ann }} v\right\rangle_{\text {int }},\left\langle\sigma_{\text {ann }} v\right\rangle_{\text {int }}\right.$ being the integral from the present temperature up to the freeze-out temperature $T_{f}$ of the thermally averaged product of the annihilation cross-section times the relative velocity of a pair of neutralinos, $x_{f}$ is defined as $x_{f} \equiv \frac{m_{\chi}}{T_{f}}$ and $g_{\star}\left(x_{f}\right)$ denotes the relativistic degrees of freedom of the thermodynamic bath at $x_{f}$. For $\left\langle\widetilde{\sigma_{\text {ann }} v}\right\rangle$ we will use the standard expansion in $\mathrm{S}$ and $\mathrm{P}$ waves: $\left\langle\widetilde{\sigma_{\text {ann }} v}\right\rangle \simeq \tilde{a}+\frac{1}{2 x_{f}} \tilde{b}$.

A lower bound on $m_{\chi}$ is now derived from Eq. (3.1.1), by requiring that

$$
\Omega_{\chi} h^{2} \leq\left(\Omega_{C D M} h^{2}\right)_{\max }
$$

In the regime of small $m_{\chi}\left(m_{\chi} \lesssim 45 \mathrm{GeV}\right)$ approximate analytic expressions for $\left\langle\widetilde{\sigma_{a n n} v}>\right.$ can be worked out. In this case the dominant terms in $\left\langle\widetilde{\left\langle\sigma_{\text {ann }} v\right\rangle_{\text {int }}}\right.$ are the contributions due to Higgs-exchange in the $s$ channel and sfermion-exchange in the $t, u$ channels of the annihilation process $\chi+\chi \rightarrow \bar{f}+f$ (interference terms are neglected). We retain only the leading terms in each contribution. Thus, for the Higgs-exchange contribution, dominated by the S-wave annihilation into down-type fermions, we have, for any final state $\bar{f}-f$, 


$$
\begin{aligned}
& \widetilde{\left\langle\sigma_{\mathrm{ann}} v\right\rangle_{f}^{\text {Higgs }} \simeq \tilde{a}_{f}^{\text {Higgs }}} \\
& \simeq \frac{2 \pi \alpha_{e . m .}^{2} c_{f}}{\sin ^{2} \theta_{W} \cos ^{2} \theta_{W}} a_{1}^{2} a_{3}^{2} \tan ^{2} \beta\left(1+\epsilon_{f}\right)^{2} \frac{\bar{m}_{f}^{2}}{m_{W}^{2}} \frac{m_{\chi}^{2}\left[1-m_{f}^{2} / m_{\chi}^{2}\right]^{1 / 2}}{\left[\left(2 m_{\chi}\right)^{2}-m_{A}^{2}\right]^{2}}
\end{aligned}
$$

where $\alpha_{e . m}$. is the electromagnetic constant, $m_{W}$ is the mass of the $W$ boson, $c_{f}$ is a standard color factor ( $c_{f}=3$ for quarks, $c_{f}=1$ for leptons), $\bar{m}_{f}$ is the fermion running mass evaluated at the energy scale $2 m_{\chi}$ and $m_{f}$ is the fermion pole mass. $\epsilon_{f}$ is a quantity which enters in the relationship between the down-type fermion running mass and the corresponding Yukawa coupling (see Ref. [1글] and references quoted therein); in the following evaluations, $\epsilon_{f}$ is negligible, except for the bottom quark, where $\epsilon_{b} \simeq 0.2$. One easily verifies that when $\left.m_{\chi}<m_{b}, \widehat{\left\langle\sigma_{\text {ann }} v\right.}\right\rangle_{f}^{H i g g s}$ entails a relic abundance exceeding the cosmological bound.

Notice that $<\widetilde{\sigma_{a n n} v}>$ turns out to be an increasing function of $m_{\chi}$. Then, to obtain a conservative lower bound on $m_{\chi}$ from the condition of Eq. (13.2i ), we have first to evaluate an $\left(\Omega_{\chi} h^{2}\right)_{\min }$ which is obtained from Eq. $(\overline{3} \cdot \overline{1})$, by replacing $<\widetilde{\sigma_{a n n} v}>$ with its maximal value $\left(\left\langle\widetilde{\sigma_{a n n} v}\right\rangle\right)_{\max }$, at fixed $m_{\chi}$. In the case of Higgs-exchange contributions, this

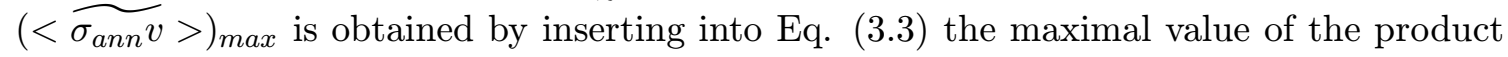

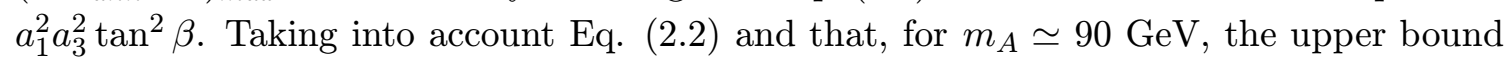
of $\tan \beta$ is $45[\underline{1} \overline{1} \overline{0}]$, we obtain $\left(a_{1}^{2} a_{3}^{2} \tan ^{2} \beta\right)_{\max } \simeq 2.6 \times 10^{2}$, and in turn

$$
\begin{aligned}
& \left(\Omega_{\chi} h^{2}\right)_{\min }^{\text {Higgs }} \equiv \\
& \frac{1.5 \cdot 10^{-10}}{\mathrm{GeV}^{2}} \frac{x_{f}}{g_{\star}\left(x_{f}\right)^{1 / 2}} \frac{m_{W}^{2}}{m_{\chi}^{2}}\left[\left(2 m_{\chi}\right)^{2}-m_{A}^{2}\right]^{2}\left(\sum_{f} \bar{m}_{f}^{2}\left(1+\epsilon_{f}\right)^{2} c_{f}\left[1-m_{f}^{2} / m_{\chi}^{2}\right]^{1 / 2}\right)^{-1} \\
& \simeq \frac{5 \cdot 10^{-11}}{\mathrm{GeV}^{2}} \frac{x_{f}}{g_{\star}\left(x_{f}\right)^{1 / 2}} \frac{m_{W}^{2}}{\bar{m}_{b}^{2}} \frac{1}{\left(1+\epsilon_{b}\right)^{2}} \frac{\left[\left(2 m_{\chi}\right)^{2}-m_{A}^{2}\right]^{2}}{m_{\chi}^{2}\left[1-m_{b}^{2} / m_{\chi}^{2}\right]^{1 / 2}}
\end{aligned}
$$

In the last step of this equation we have included only the dominant contribution due to the $b-\bar{b}$ final state.

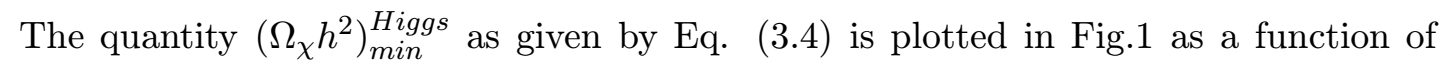
$m_{\chi}$, for the value $m_{A}=90 \mathrm{GeV}$ (current experimental lower bound). The two horizontal lines denote two representative values for the upper bound on $\Omega_{C D M} h^{2}:\left(\Omega_{C D M} h^{2}\right)_{\max }=$ 0.3 (short-dashed line) and $\left(\Omega_{C D M} h^{2}\right)_{\max }=0.131$ (long-dashed line). Fig.1 displays how a lower bound on $m_{\chi}$ is derived from an upper limit on $\Omega_{C D M} h^{2}$. In particular, using $T_{Q C D}=300 \mathrm{MeV}$, where $T_{Q C D}$ is the hadron-quark transition temperature, one obtains from $\left(\Omega_{C D M} h^{2}\right)_{\max }=0.3$ the bound $m_{\chi} \gtrsim 5.2 \mathrm{GeV}$, a value which increases to $m_{\chi} \gtrsim 6.2$ $\mathrm{GeV}$, when the value $\left(\Omega_{C D M} h^{2}\right)_{\max }=0.131$ is employed. Finally, to support the validity of the analytical approximations employed to derive Eq. (13.4), in Fig.1 we also display the scatter plot of $\Omega_{\chi} h^{2}$, when a numerical scanning of the supersymmetric parameter space is performed. From our previous formulae one obtains that the bound on $m_{\chi}$ simply scales with $m_{A}$ as follows: 


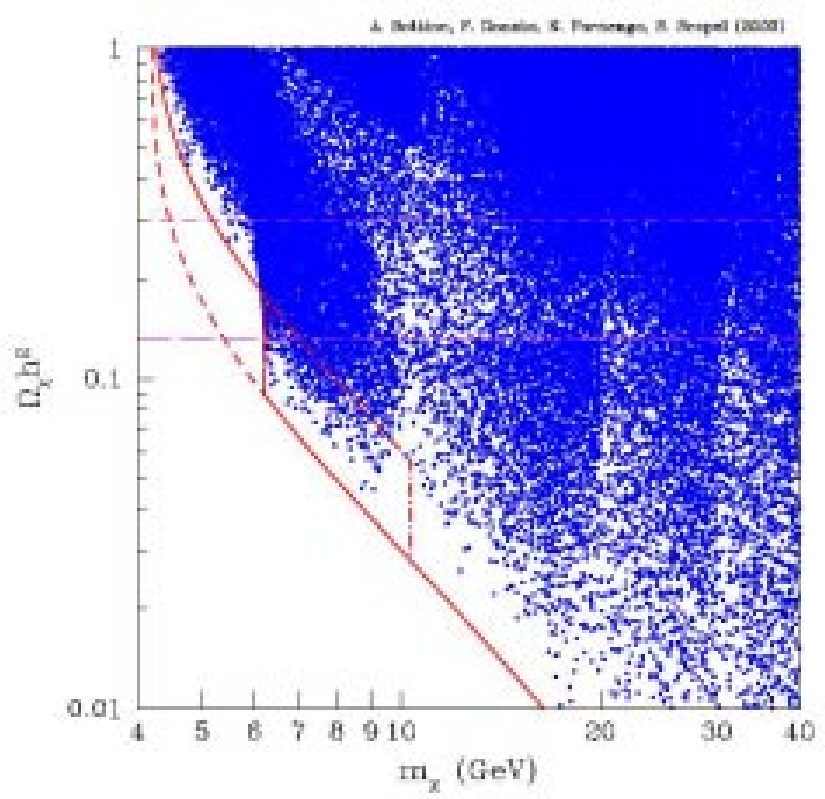

Figure 1: Neutralino relic abundance $\Omega_{\chi} h^{2}$ as a function of the mass $m_{\chi}$. The solid curve

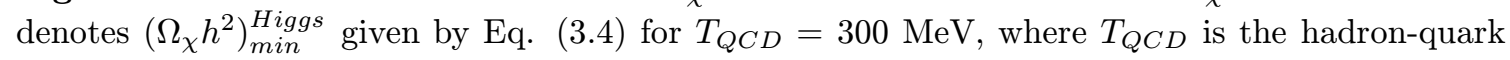
transition temperature. Dashed and dot-dashed curves refer to the representative values $T_{Q C D}=$ $100 \mathrm{MeV}, T_{Q C D}=500 \mathrm{MeV}$, respectively. The two horizontal lines denote two representative values of $\Omega_{C D M} h^{2}: \Omega_{C D M} h^{2}=0.3$ (short-dashed line) and $\Omega_{C D M} h^{2}=0.131$ (long-dashed line). The scatter plot is obtained by a full scanning of the supersymmetric parameter space.

$$
m_{\chi}\left[1-m_{b}^{2} / m_{\chi}^{2}\right]^{1 / 4} \gtrsim 5.3 \mathrm{GeV}\left(\frac{m_{A}}{90 \mathrm{GeV}}\right)^{2}
$$

For $m_{A} \gtrsim 200-300 \mathrm{GeV}$, the annihilation cross section of light neutralinos is dominated by sfermion-exchange. With the aid of numerical evaluations, it is found that the leading contributions to $\left\langle\widetilde{\left.\sigma_{\text {ann }} v\right\rangle}\right.$ sfermion $\equiv \sum_{f}\left\langle\widetilde{\sigma_{\text {ann }} v}\right\rangle_{f}^{\text {sfermion }}$ are provided by the term due to the $\tau$ lepton: $\left\langle\widetilde{\sigma_{\text {ann }} v}\right\rangle^{\text {sfermion }} \simeq\left\langle\widetilde{\sigma_{\text {ann }} v}\right\rangle_{\tau}^{\text {sfermion }}$. This is in turn maximized by (see Ref. $[\overline{i n}$ in for details):

$$
\left({\widetilde{\sigma_{\text {ann }} v}}^{\text {sfermion }}\right)_{\max } \simeq \frac{\pi \alpha_{e . m .}^{2}}{8 \cos ^{4} \theta_{W}} \frac{m_{\chi}^{2}\left[1-m_{\tau}^{2} / m_{\chi}^{2}\right]^{1 / 2}}{m_{\tilde{\tau}^{4}}^{4}}\left[\left(2+\frac{5}{2} \frac{m_{\tau}}{m_{\chi}}\right)^{2}+\frac{23}{2 x_{f}}\right]
$$

Assuming $m_{\tilde{\tau}}=87 \mathrm{GeV}$ (current experimental lower bound) one finds in this case a lower bound for the neutralino mass $m_{\chi} \gtrsim 14 \mathrm{GeV}$ for $\left(\Omega_{C D M} h^{2}\right)_{\max }=0.3$, and $m_{\chi} \gtrsim 22$ $\mathrm{GeV}$ for $\left(\Omega_{C D M} h^{2}\right)_{\max }=0.131$. The scaling of this last bound with the stau mass is approximately given by 


$$
m_{\chi}\left[1-m_{\tau}^{2} / m_{\chi}^{2}\right]^{1 / 4} \gtrsim 22 \mathrm{GeV}\left(\frac{m_{\tilde{\tau}}}{90 \mathrm{GeV}}\right)^{2} .
$$

This limit is compatible with the results of Refs. [i2 $\overline{2} \overline{0}]-\left[2 \overline{1}_{1}^{\prime}\right]$, where the lower bounds $m_{\chi} \gtrsim(15-18) \mathrm{GeV}$ are found assuming that $m_{A}$ is very large $\left(m_{A} \sim 1 \mathrm{TeV}\right)$.

\section{Detectability of light neutralinos by WIMP direct measurements}

The neutralino configurations which provide the highest values of direct detection rates are the ones dominated by $(h, H)$ Higgs-exchange processes, which in turn require a gauginohiggsino mixing. Under these hypotheses, using the expression of $\Omega_{\chi} h^{2}$ given above and an analytical approximation also for $\sigma_{\text {scalar }}^{\text {(nucleon) }}$ (see Ref. [i2i]) we obtain the following relation between the scalar neutralino-nucleon cross-section and the neutralino relic abundance:

$$
\begin{aligned}
& \left(\Omega_{\chi} h^{2}\right) \sigma_{\text {scalar }}^{\text {(nucleon })} \simeq \\
& 1.4 \times 10^{-40} \mathrm{~cm}^{2} \mathrm{~T}\left(\frac{m_{s}\langle N|\bar{s} s| N\rangle}{200 \mathrm{MeV}}\right)^{2} \frac{\mathrm{GeV}^{2}}{m_{\chi}^{2}\left[1-m_{b}^{2} / m_{\chi}^{2}\right]^{1 / 2}}\left(\frac{m_{A}}{m_{h}}\right)^{4},
\end{aligned}
$$

where $m_{h}$ is the mass of the lightest CP-even neutral Higgs boson, $T$ is given by

$$
T=\frac{\left(a_{3} \sin \alpha+a_{4} \cos \alpha\right)^{2}}{\left(a_{4} \cos \beta-a_{3} \sin \beta\right)^{2}} \frac{\left(\sin \alpha+\epsilon_{s} \cos (\alpha-\beta) \sin \beta\right)^{2}}{\sin ^{2} \beta\left(1+\epsilon_{b}\right)^{2}} .
$$

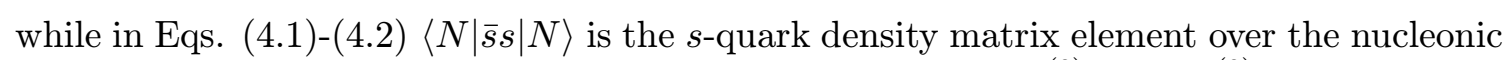
state. Here $\alpha$ is the angle which rotates the Higgs fields $H_{1}^{(0)}$ and $H_{2}^{(0)}$ into the mass

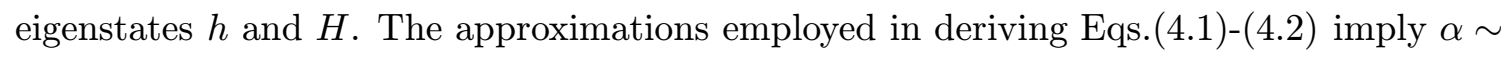
$\frac{\pi}{2}, m_{h} \sim m_{A} \sim 100 \mathrm{GeV}$, so that $T$ is of order one.

Thus, for neutralino configurations with $m_{\chi} \lesssim 20 \mathrm{GeV}, \sigma_{\text {scalar }}^{(\text {(nucleon })}$ turns out to be bounded by

$$
\sigma_{\text {scalar }}^{\text {(nucleon) }} \gtrsim \frac{10^{-40} \mathrm{~cm}^{2}}{\left(\Omega_{C D M} h^{2}\right)_{\max }} \frac{\mathrm{GeV}^{2}}{m_{\chi}^{2}\left[1-m_{b}^{2} / m_{\chi}^{2}\right]^{1 / 2}} .
$$

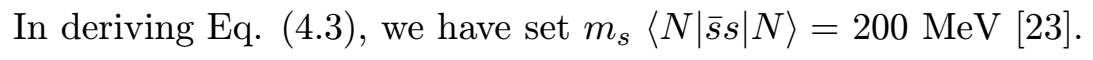

A complete numerical evaluation of the neutralino-nucleon cross section, where all relevant diagrams are taken into account, is displayed in Fig.2. Here the quantity $\xi \sigma_{\text {scalar }}^{\text {(nucleon }}$ is plotted as a function of the neutralino mass, where $\xi$ is a rescaling factor between the neutralino local matter density $\rho_{\chi}$ and the total local dark matter density $\rho_{0}: \xi \equiv \rho_{\chi} / \rho_{0}$ (following a standard assumption, $\xi$ may be taken as $\xi=\min \left(1, \Omega_{\chi} h^{2} /\left(\Omega_{C D M} h^{2}\right)_{\min }\right)$, where we assume $\left(\Omega_{C D M} h^{2}\right)_{\min }=0.095$, as defined in the previous section). The peculiar funnel in the scatter plot for $m_{\chi} \lesssim 20 \mathrm{GeV}$ is due to the bound of Eq. (4. the contour line delimits the annual modulation region recently derived by the DAMA

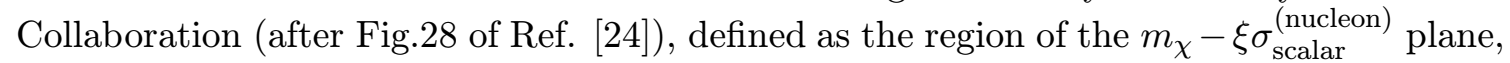
where the likelihood-function values are distant more than $4 \sigma$ from the null (absence of modulation) hypothesis. In deriving this contour line, the DAMA Collaboration has taken 

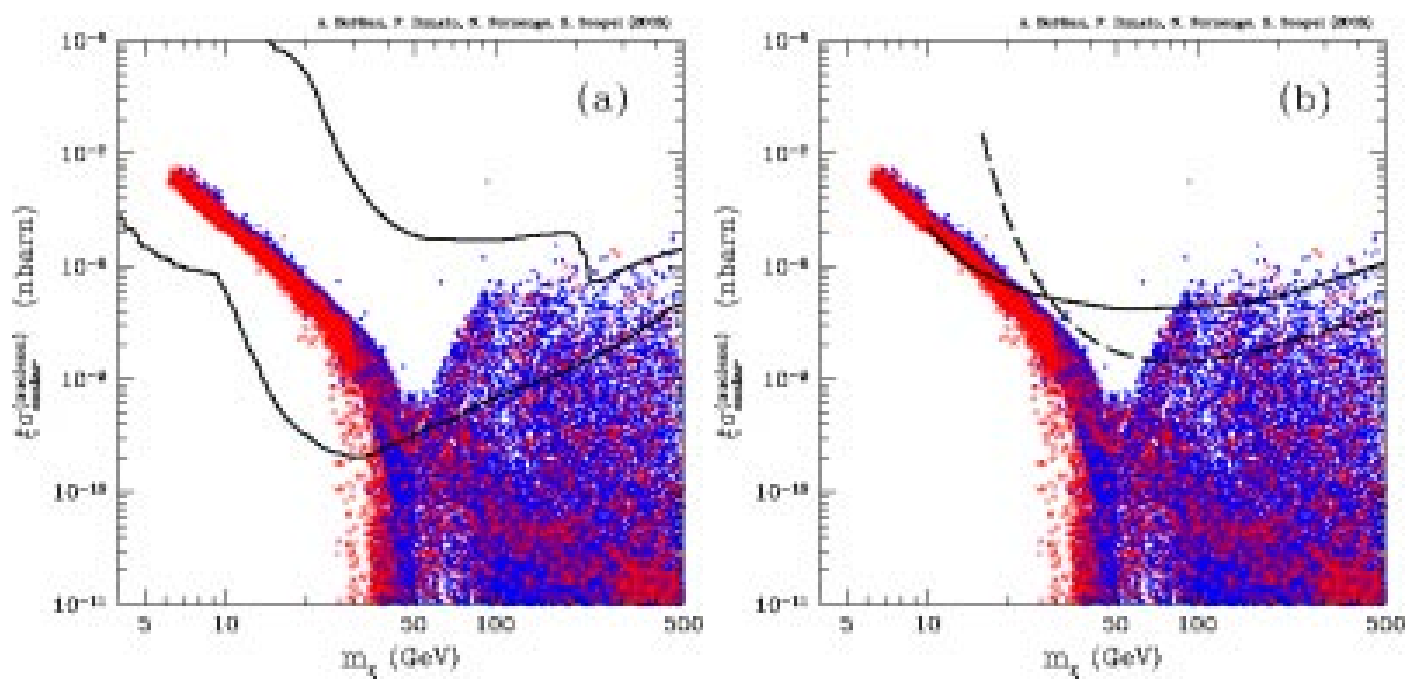

Figure 2: Scatter plot of $\xi \sigma_{\text {scalar }}^{\text {(nucleon) }}$ versus $m_{\chi}$. Crosses (red) and dots (blue) denote neutralino configurations with $\Omega_{\chi} h^{2} \geq\left(\Omega_{C D M} h^{2}\right)_{\min }$ and $\Omega_{\chi} h^{2}<\left(\Omega_{C D M} h^{2}\right)_{\min }$, respectively $\left(\left(\Omega_{C D M} h^{2}\right)_{\min }=\right.$ 0.095) (a) The curves delimit the DAMA region where the likelihood-function values are distant more than $4 \sigma$ from the null (absence of modulation) hypothesis [2] 24$]$; this region is the union of the regions obtained by varying the WIMP DF over the set considered in Ref. [25in]. (b) The solid and the dashed lines are the experimental upper bounds given by the CDMS [26] and the EDELWEISS [27-7] Collaborations, respectively, under the hypothesis that the WIMP DF is given by an isothermal distribution with a standard set of astrophysical parameters.

into account a rather large class of possible phase-space distribution functions (DF) for WIMPs in the galactic halo[25 $\left.2 \overline{5}_{1}\right]$. From Fig.2(a) we derive that the entire population of relic neutralinos with $m_{\chi} \lesssim 25 \mathrm{GeV}$ as well as a significant portion of those with a mass up to about $50 \mathrm{GeV}$ are within the annual-modulation region of the DAMA Collaboration.

Other experiments of WIMP direct detection have recently published new data: CDMS[2] $\left.\overline{2}_{\mathbf{L}}\right]$ and EDELWEISS[2 $\left.\overline{2}_{2}\right]$. Both results are given in terms of an upper bound on $\sigma_{\text {scalar }}^{\text {(nucleon }}$ for a given DF (an isothermal distribution) and for a single set of the astrophysical parameters: $\rho_{0}=0.3 \mathrm{GeV} \cdot \mathrm{cm}^{-3}, v_{0}=220 \mathrm{~km} \cdot \mathrm{s}^{-1}$ ( $v_{0}$ is the local rotational velocity). These upper bounds are displayed in Fig.2(b), where it is possible to see that in case of an isothermal $D F$ with the representative values of parameters given above, a sizeable subset of supersymmetric configurations in the mass range $10 \mathrm{GeV} \lesssim m_{\chi} \lesssim 20 \mathrm{GeV}$ would be incompatible with the CDMS experimental upper bound (together with some configurations with $m_{\chi} \gtrsim$ $80 \mathrm{GeV}$ ), while the EDELWEISS constraint turns out to be marginal for the low-mass population.

However, in order to set solid constraints on theoretical predictions, astrophysical uncertainties should not be neglected. In order to quantify these uncertainties, in Figs. we show, for different choices of the WIMP velocity distribution DF, the expected energy spectrum for direct detection on a Sodium Iodide target and a Germanium one. In both figures the left-hand side shows the time-averaged component, while the right-hand one the modulation amplitude, defined as the difference between the signal expected in June and that expected in December (divided by two). In both figures the neutralino parameters 

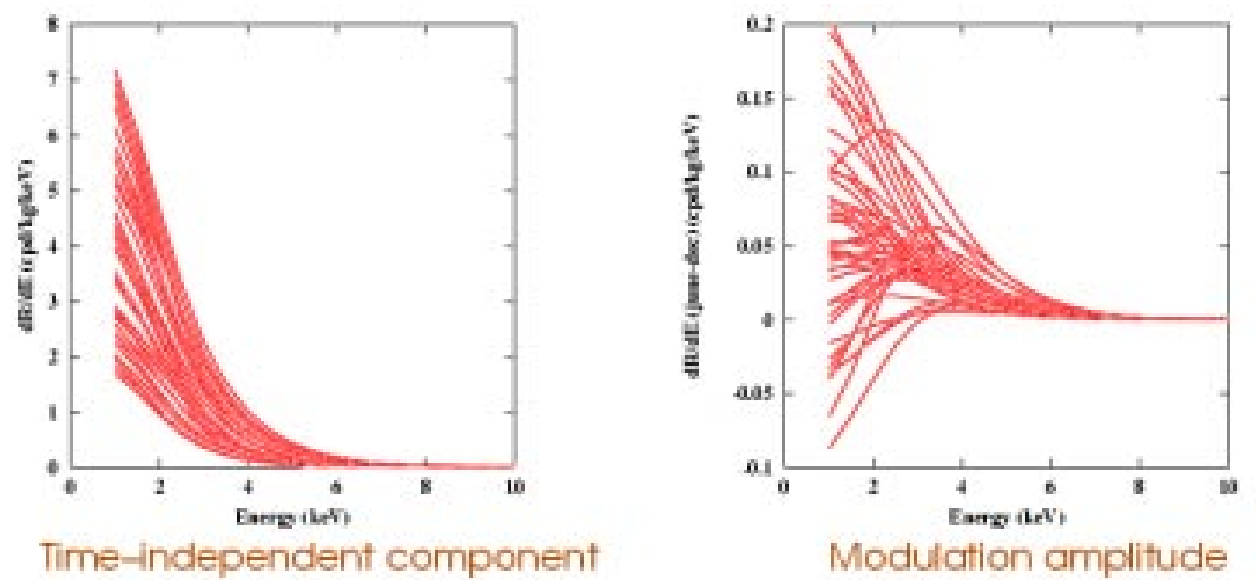

Figure 3: Example calculation of the constant (left) and modulated (right) components of the expected WIMP energy spectrum in a Sodium Iodide detector for $m_{\chi}=50 \mathrm{GeV}$ and $\sigma_{\text {scalar }}^{\text {(nucleon }}=10^{-8}$ nbarn. In both panels each curve corresponds to one of the halo models listed in Table II of Ref. [25i].

are fixed to the representative values $m_{\chi}=50 \mathrm{GeV}$ and $\sigma_{\text {scalar }}^{\text {(nucleon }}=10^{-8}$ nbarn, while each curve refers to one of the models for DF listed in Table II of Ref. [205i] the significant uncertainties introduced by astrophysics in theoretical predictions.
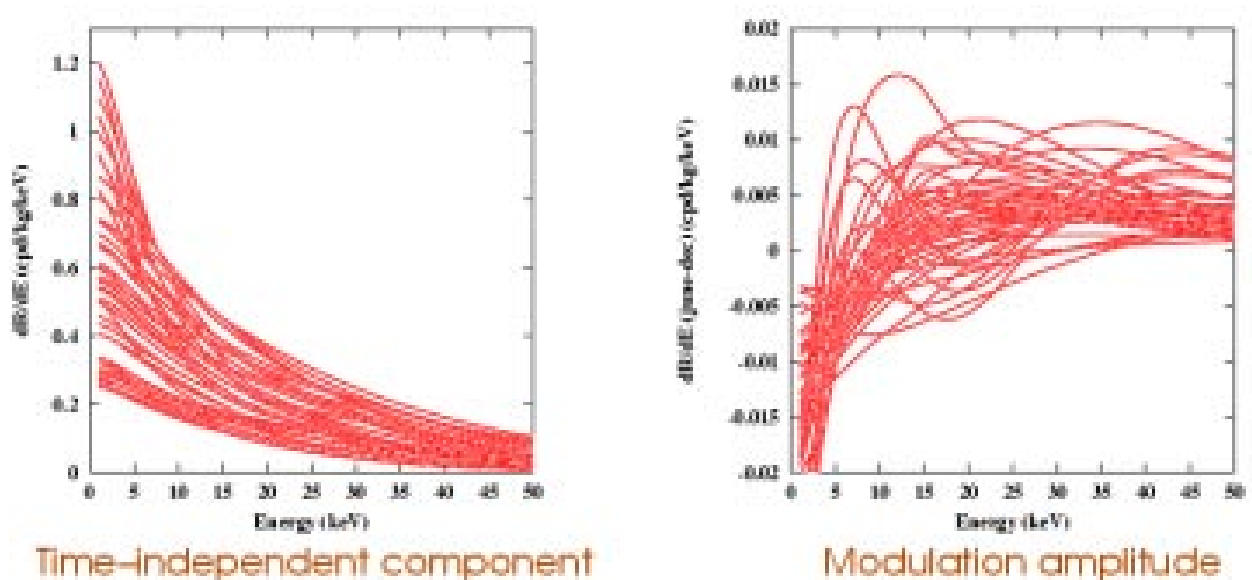

Figure 4: The same as in Fig. $\underline{31}_{1}$ for a germanium target.

As a further example of the impact of the choice of a particular velocity distribution function on theoretical expectations, in Fig. ${ }_{-1}^{\prime} \bar{\prime}$ ( taken from Ref. $\left.[\overline{2} \overline{2} \overline{9}]\right)$ the quantity $\mathcal{I}\left(v_{\min }\right) \equiv$ $\int_{w \geq v_{\min }} d \vec{w} \frac{f_{\mathrm{ES}}(\vec{w})}{w}$, which factorizes the dependence of the direct detection differential rate $d R / d E_{R}$ on the velocity distribution function, is plotted as a function of time (in days). Here $f_{\mathrm{ES}}$ is the WIMP velocity distribution function in the Earth's rest frame, and is assumed to be triaxial in the Galactic rest frame: $f_{\mathrm{GAL}}=N \exp \left(-\frac{v_{x}^{2}}{2 \sigma_{x}^{2}}-\frac{v_{y}^{2}}{2 \sigma_{y}^{2}}-\frac{v_{z}^{2}}{2 \sigma_{z}^{2}}\right)$, with $\sigma_{x} / \sigma_{y}=0.2$ and $\sigma_{z} / \sigma_{y}=0.8 ; w$ is the WIMP velocity in the Earth's rest frame; $v_{\min }$ is the minimum value of $w$ for a given recoil energy $E_{R}$, WIMP mass $m_{W}$ and nuclear target mass $m_{N}$, and is given by: $v_{\text {min }} \equiv \sqrt{E_{R} /\left(2 m_{N}\right)}\left(m_{W}+m_{N}\right) / m_{W}$. From this figure one can see that also the sinusoidal time variation of the expected WIMP direct detection rate 


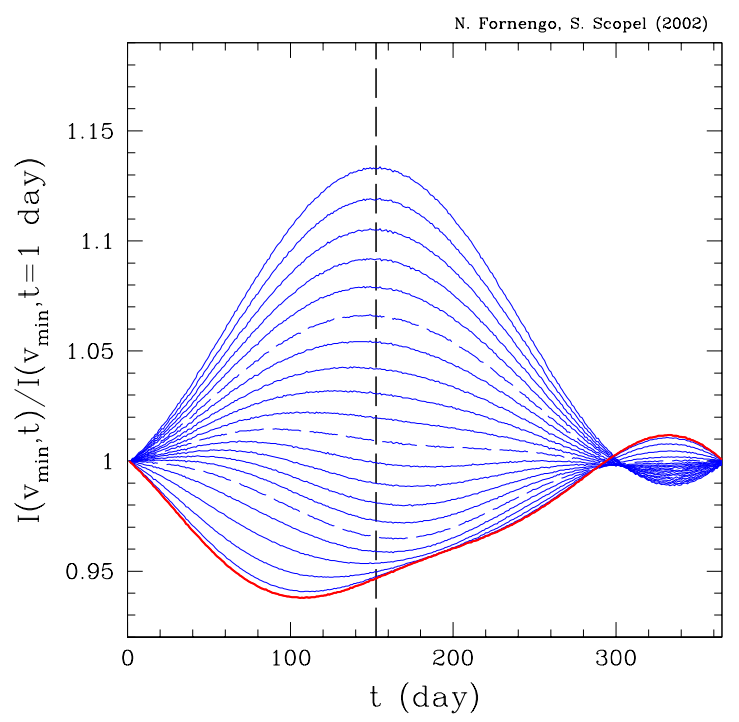

Figure 5: Expected WIMP direct detection rate (in arbitrary units) as a function of time (in days) during one year, and for an anisotropic model for the velocity distribution function (see text). The different curves correspond to different recoil energies. The vertical dashed line denotes $t=152$ days, when the relative velocity between the Earth and the WIMP halo is expected to be maximal.

during the year, which is universally assumed in the analysis on annual modulation, can be distorted in the case of a departure from the usual Maxwellian distribution.

From the present discussion we conclude that, in order to derive upper bounds on $\xi \sigma_{\text {scalar }}^{\text {(nucleon }}$ from the experimental data in a reliable way, it would be necessary to consider a large variety of DFs and of their corresponding astrophysical parameters (with their own uncertainties); the intersection of these bounds would provide an absolute limit to be used to possibly exclude a subset of the supersymmetric parameter space. A combined investigation of all experiments along these lines is not available at the moment.

In conclusion, the experimental exploration of the low-mass neutralino population is already under way in case of some experiments of WIMP direct detection and within the reach of further investigation in the near future. We just mention that, as far as indirect neutralino searches are concerned, current data from $\bar{p}$ 's, $\gamma$ 's and up-going $\mu$ 's, if interpreted conservatively, do not yet set significant constraints on these configurations [푸으.

\section{Acknowledgments}

This talk is based on work done in collaboration with A. Bottino, F. Donato and N. Fornengo. 


\section{References}

[1] K. Hagiwara et al., Phys. Rev. D 66, 010001 (2002).

[2] A. Bottino, N. Fornengo and S. Scopel, Phys. Rev. D 67, 063519 (2003).

[3] A. Bottino, F. Donato, N. Fornengo and S. Scopel, Phys. Rev. D 68, 043506 (2003).

[4] A. Bottino, F. Donato, N. Fornengo and S. Scopel, arXiv:hep-ph/0307303.

[5] D.N. Spergel et al. (WMAP), arXiv:astro-ph/0302209.

[6] T. J. Pearson et al. (CBI), Astrophys. J. 591, 556 (2003).

[7] C.L. Kuo et al. (ACBAR), arXiv:astro-ph/0212289.

[8] A. Bottino, F. Donato, N. Fornengo and S. Scopel, Phys. Lett. B423, 109 (1998); Phys. Rev. D 59, 095003 (1999); Phys. Rev. D 59, 095004 (1999); Phys. Rev. D 62, 056006 (2000).

[9] A. Colaleo (ALEPH Collaboration), talk at SUSY'01), June 11-17, 2001, Dubna, Russia; J. Abdallah et al. (DELPHI Collaboration), DELPHI 2001-085 CONF 513, June 2001.

[10] T. Affolder at al., Phys. Rev. Lett. 86, 4472 (2001).

[11] S. Ahmed et al., (CLEO Collaboration), CONF 99/10, arXiv:hep-ex/9908022; R. Barate et al. (ALEPH Collaboration), Phys. Lett B 429, 169 (1998); K. Abe et al. (Belle Collaboration), Phys. Lett. B 511, 151 (2001).

[12] A. Bottino, N. Fornengo, S. Scopel, Nucl. Phys. B608, 461 (2001).

[13] A. Bottino, F. Donato, N. Fornengo, S. Scopel, Phys. Rev. D63, 125003 (2001).

[14] S. Ambrosanio, A. Dedes, S. Heinemeyer, S. Su, G. Weiglein, Nucl. Phys. B624, 3 (2002).

[15] G.W. Bennett et al. (Muon $(g-2)$ Collaboration), Phys. Rev. Lett. 89, 101804 (2002); Erratum-ibid. 89, 129903 (2002).

[16] M. Davier, S. Eidelman, A. Hocker and Z. Zhang, Eur. Phys. J. C 27, 497 (2003).

[17] K. Hagiwara, A. D. Martin, D. Nomura and T. Teubner, Phys. Lett. B 557, 69 (2003).

[18] W.J. Percival et al., MNRAS 327, 1297 (2001).

[19] R.A.C. Croft et al., ApJ 581, 20 (2002); N.Y. Gnedin and A.J.S. Hamilton, MNRAS 334, 107 (2002).

[20] G. Bélanger, F. Boudjema, A. Pukhov and S. Rosier-Lees, arXiv:hep-ph/0212227.

[21] D. Hooper and T. Plehn, Phys. Lett. B 562, 18 (2003).

[22] A. Bottino, F. Donato, N. Fornengo and S. Scopel, Astropart. Phys. 13, 215 (2000).

[23] A. Bottino, F. Donato, N. Fornengo, S. Scopel Astropart. Phys. 18, 205 (2002).

[24] R. Bernabei et al., Riv. N. Cim. 26 n. 1 (2003) 1-73.

[25] P. Belli, R. Cerulli, N. Fornengo and S. Scopel, Phys. Rev. D 66, 043503 (2002).

[26] D.S. Akerib et al., arXiv:hep-ex/0306001.

[27] A. Benoit et al., Phys. Lett. B 545, 43 (2002).

[28] A. Bottino, F. Donato, N. Fornengo, S. Scopel, Phys. Lett. B 423, 109 (1998); Phys. Rev. D 62, 056006 (2000); Phys. Rev. D 63, 125003 (2001).

[29] N. Fornengo and S. Scopel, Phys. Lett. B 576, 189 (2003).

[30] N. Fornengo, these Proceedings. 